\title{
Frecuencia de maloclusiones sagitales y transversales en estudiantes de 12 a 18 años de Asunción
}

\author{
Carlos Rafael I nvernizzi-Mendoza , Lourdes Valdez-Godoy , Cristina Raquel Caballero- \\ García , Mirna Santander-Aguilera , Petrona Benítez-Torres , Liliana Cardozo-Vera \\ Francisco Flores-Romero , Claudia Alcaraz-Castillo \\ ${ }^{1}$ Universidad Autónoma de Asunción, Facultad de Odontología. Asunción, Paraguay
}

Cómo referenciar este artículo/ How to reference this article:

Invernizzi-Mendoza CR, Valdez-Godoy L, Caballero-Garcia CR, Santander-Aguilera $M$, Benítez Torres P, Cardozo-Vera L, Flores Romero F, Alcaraz-Castillo C. Frecuencia de maloclusiones sagitales y transversales en estudiantes de 12 a 18 años de Asunción. Mem. Inst. Investig. Cienc. Salud. 2020; 18(3): 17-23

\section{RE S U M E N}

La maloclusión es uno de los trastornos bucodentales más comunes. Reconocer su importancia lleva a la necesidad de realizar estudios epidemiológicos cuyos resultados orienten el desarrollo de programas para su prevención, intercepción y tratamiento. EI objetivo de la presente investigación fue determinar la frecuencia de maloclusión en sentido sagital y transversal en estudiantes de nivel secundario de la ciudad de Asunción. Se realizó un estudio descriptivo de corte transversal. La población de estudio estuvo conformada por los alumnos de 12 a 18 años inscriptos en colegios públicos y privados de la ciudad de Asunción durante el 2017. Los datos recabados de la inspección bucodental fueron anotados en fichas clínicas yanalizadas con el programa Epi-Info TM. El número de participantes fue de 1047 estudiantes que cumplieron con los criterios de inclusión. El $52 \%$ de los participantes fueron de sexo femenino y el $48 \%$ del sexo masculino. La frecuencia de maloclusión en sentido sagital fue del $98 \%$, y en sentido transversal del 27,6\%. Entre las maloclusiones en sentido sagital la más frecuente fue la Clase I (53\%). La frecuencia de mordida cruzada fue del $23 \%$, de los cuales, el $43 \%$ presentó mordida cruzada anterior y el $57 \%$ mordida cruzada posterior. De los que presentaron mordida cruzada posterior, el $61 \%$ fue unilateral y el $39 \%$ bilateral.Se observa una importante frecuencia de maloclusiones en la población de estudio indicativa de la necesidad de establecer acciones para su diagnóstico y tratamiento oportunos.

Palabras clave: Maloclusión, mordida cruzada, clasificación de Angle.

\section{Frequency of sagittal and transverse malocclusions in students from 12 to 18 years of age in Asunción}

\begin{abstract}
A B S T R A C T
Malocclusion is one of the most common oral disorders. Recognizing its importance leads to the need for epidemiological studies whose results guide the development of programs for its prevention, interception and treatment. The objective of the present investigation was to determine the frequency of malocclusion in the sagittal and transverse direction in secondary school students from the city of Asunción. A descriptive cross-sectional study was carried out. The study population was made up of students from 12 to 18 years old enrolled in public and private schools in the city of Asunción during 2017. The data collected from the oral inspection was recorded in clinical records and analyzed with the Epi-Info program. TM. The number of participants was 1047 students who met the inclusion criteria. Fifty two percent of the
\end{abstract}

Fecha de recepción: Junio2020. Fecha de aceptación: Octubre 2020

* Autor correspondiente: Carlos Rafael Invernizzi-Mendoza. Universidad Autónoma de Asunción. Asunción, Paraguay

Email: carlosinvernizzi@hotmail.com 
participants were female and $48 \%$ male. The frequency of malocclusion in the sagittal direction was $98 \%$, and in the transverse direction it was $27.6 \%$.Among sagittal malocclusions, the most frequent was Class I (53\%). The frequency of crossbite was $23 \%$, of which $43 \%$ presented an anterior crossbite and $57 \%$ a posterior crossbite. Of those who presented posterior crossbite, $61 \%$ were unilateral and $39 \%$ bilateral. An important frequency of malocclusions is observed in the study population indicative of the need of establishing actions for its timely diagnosis and treatment.

Keywords: Malocclusion, cross bite, Angle's classification.

\section{NTRODUCCI ÓN}

La maloclusión es la alteración del crecimiento y desarrollo normal de los dientes, loscomponentes óseos y musculares, producto de una compleja interacción de factores. Esto afecta al sistema estomatognático y altera las funciones masticatorias, de fonación y deglución, sin olvidar que además afecta la estética del paciente y su calidad de vida(1-5)

La maloclusión afecta a un amplio sector de la población, y según datos que brinda la Organización Mundial de la Salud (OMS), esta alteración constituye la tercera afección bucal de mayor prevalencia a nivel mundial, después de la caries y la enfermedad periodontal ${ }^{(6)}$. En 1899, Angle ideó un esquema simple y mundialmente aceptado para clasificar la maloclusión en sentido sagital, es el método más utilizado para evaluar la relación oclusal. Esta clasificación se basa en la posición de la cúspide mesio vestibular del primer molar superior y su relación anteroposterior con el surco mesio vestibular del primer molar inferior. Angle dividió la maloclusión en tres grandes grupos: Clase I, Clase II, Clase III ${ }^{(3,7,8)}$.

Entre las maloclusiones en sentido transversal existe un tipo de maloclusión muy frecuente denominado mordida cruzada, que puede ser anterior o posterior. La mordida cruzada anterior es una anomalía de la oclusión en el plano anteroposterior caracterizada por la presencia de un resalte negativo donde se invierte la relación de desbordamiento de los dientes superiores por fuera de los inferiores ${ }^{(9-13)}$.

La mordida cruzada posterior se presenta cuando hay una relación anormal labiolingual o bucolingual de los dientes y en la mayoría de los casos es el resultado de un estrechamiento bilateral del maxilar. Se puede presentar una mordida cruzada posterior unilateral, cuando afecta a solo un lado y bilateral cuando afecta a ambos lados de la arcada ${ }^{(14,15)}$. Esta patología ha sido relacionada con muchos hábitos que podrían ser sus factores etiológicos, aunque las actuales investigaciones no son determinantes, los más habituales son la succión digital, la deglución atípica y la respiración bucal ${ }^{(16,17)}$.

Otra alteración en sentido transversal es la mordida en tijera, que es una alteración bastante rara y su prevalencia en la población es menor al 1\%. El causante de esta alteración suele ser el maxilar inferior, debido a una marcada inclinación de los dientes posteroinferiores hacia lingual o debido a una asimetría mandibular. Si todas estas anomalías no son tratadas a tiempo, pueden generar patologías en la articulación temporomandibular, favorecer un crecimiento anormal del maxilar inferior y generar una asimetría en los maxilares ${ }^{(8,19)}$.

La detección temprana de maloclusiones en edad de desarrollo es crítica para evitar intervenciones terapéuticas invasivas y permite planificar tratamientos interceptivos que sean costo efectivos para los pacientes. El objetivo del presente trabajo fue determinar la frecuencia de maloclusión en sentido sagital y transversal en estudiantes de 12 a 18 años de colegios públicos y privados de la ciudad de AsunciónParaguay en el 2017, así como también sus respectivas variaciones clínicas.

\section{MATERI ALES Y MÉTODOS}

Estudio descriptivo observacional de corte transversal. Se realizó un tipo de muestreo no probabilístico de casos consecutivos. Se incluyó a todos los alumnos de 12 a 18 años inscriptos en colegios públicos y privados de la ciudad de AsunciónParaguay que fueron seleccionados por conveniencia, en el 2017.Fueron excluidos los adolescentes con tratamiento ortodóntico, los que no presentaron el consentimiento 
informado firmado por sus padres y los que rechazaron participar en la investigación. La recolección de los datos se realizó mediante inspección bucodental en fichas clínicas completadas por los estudiantes del último año de la carrera de odontología de la Universidad Autónoma de Asunción bajo la supervisión de los docentes de la cátedra de ortodoncia. La clasificación de Angle ${ }^{(20)}$ fue utilizada para determinar la relación oclusal. Los datos fueron analizados con el programa Epi-Info TM V7.1 (CDC, Atlanta, US).

La información recolectada fue manejada confidencialmente y para fines únicamente académicos. Se respetaron en todo el proceso de la investigación los tres principios básicos de la ética: el respeto por los sujetos, el principio de beneficencia y el de justicia.

\section{RESULTADOS}

Participaron 1047estudiantes entre 12 y 18 años. La Figura 1 presenta el porcentaje de estudiantes que fueron evaluados por edad. El 52\% (546) pertenecía al sexo femenino. La frecuencia de maloclusión en sentido sagital fue del $98 \%$, y en sentido transversal del $27,6 \%$.

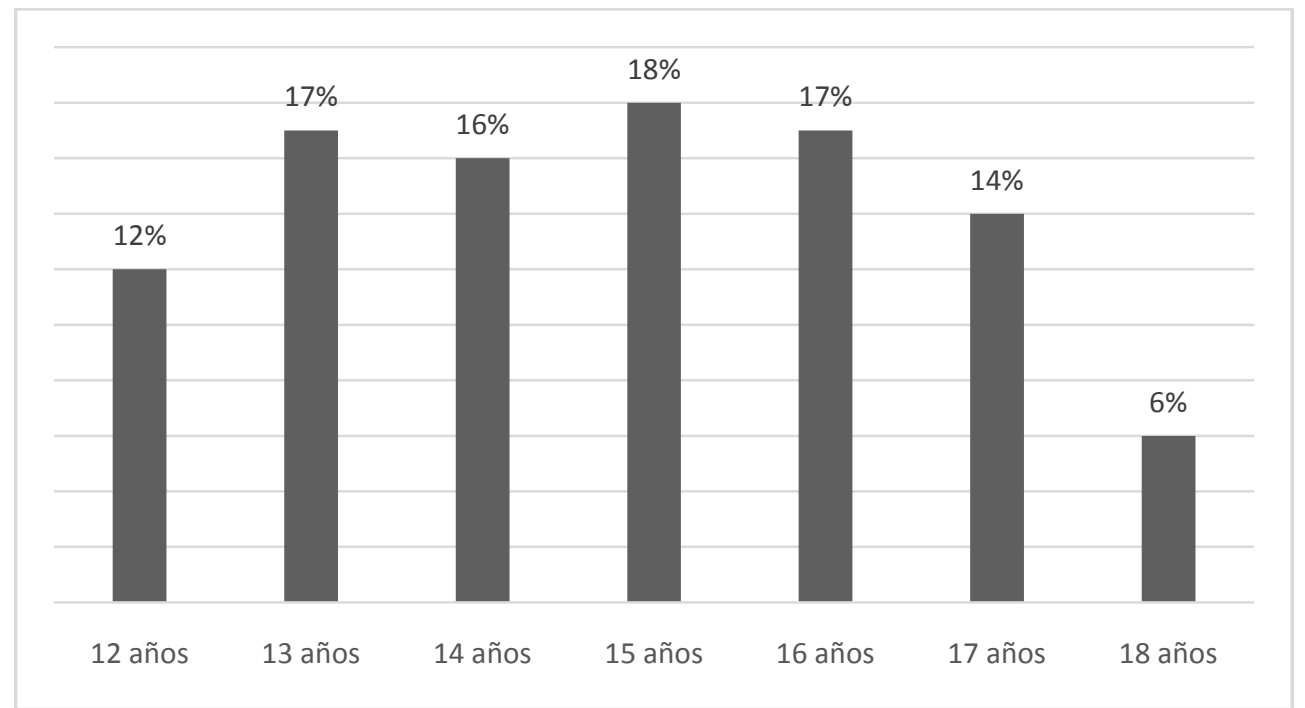

Figura 1. Frecuencia de pacientes evaluados por edad $=1047$

Entre las maloclusiones en sentido sagital la más frecuente fue la Clase I, que se presentó en un 53\% (Ver Figura 2).

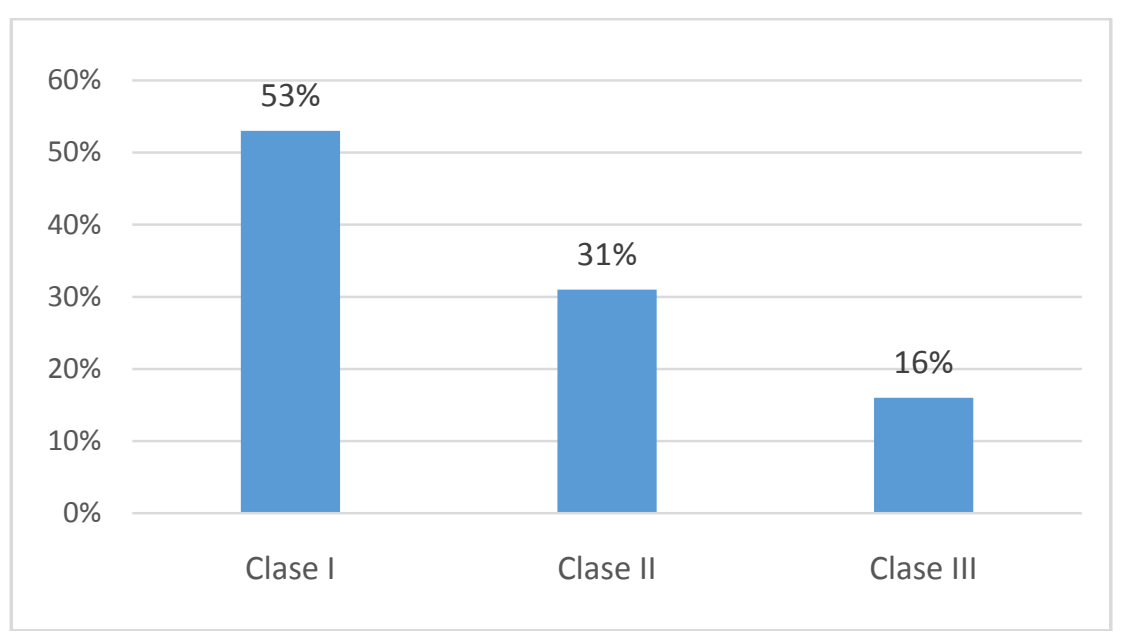

Figura 2. Frecuencia de maloclusión según la clasificación del Angle. N=1047. 
La frecuencia de mordida cruzada fue del $23 \%$, de los cuales el $43 \%$ presentó mordida cruzada anterior y el $57 \%$ mordida cruzada posterior. De los que presentaron mordida cruzada posterior, el $61 \%$ fue unilateral y el $39 \%$ bilateral.

La frecuencia de mordida en tijera fue del $5 \%$, encontrándose mordida en tijera unilateral en un $4 \%$ y bilateral en el $1 \%$ de la población.

\section{DISCUSIÓN}

Esta investigación aporta datos epidemiológicos para detectar tendencias en las maloclusiones en la edad adolescente, etapa donde se consolidan las estructuras dentales y maxilofaciales de las personas.

En el presente trabajo, según la clasificación de Angle ${ }^{(21)}$, se presentó maloclusión de Clase I en un 53\%; cifra inferior a las encontradasen otras investigaciones como la de Murrieta et. al. ${ }^{(22)}$ que reportó un $72,8 \%$ en México, Thilander ${ }^{(23)}$ que encontró un $75,5 \%$ en Bogotá-Colombia y Mafla ${ }^{(24)}$ que reportó un $68,7 \%$ en Pasto-Colombia. También reportaron resultados superiores Fajardo ${ }^{(25)}$ en Ecuador con un $72 \%$, Rodríguez ${ }^{(26)}$ en Trujillo-Perú con un $69 \%$, Medina ${ }^{(27)}$ en Caracas-Venezuela con un $64 \%$ y Boeck ${ }^{(28)}$ en Araquara-Brasil, con un $69 \%$. Por el contrario, reportan cifras menores al $53 \%$ de Clase I identificado en la población de este estudio, Cifuentes ${ }^{(29)}$ et. al. en Chilequienes encontraron $45 \%$ y Castillo ${ }^{(30)}$ et. al.en NayaritMéxicoque observaron46\% de pacientes Clase I.

Con respecto a la Clase II, en el presente trabajo se identificó en un 31\%, cifra similar a la reportada por Cifuentes $(37 \%)$, Castillo $(30 \%)$ y Boeck $(27 \%)^{(28-30)}$. Por otro lado, la frecuencia de pacientes con clase II resultó superior a la encontrada por investigacionesrealizadas en otros países de la región como Colombia ( $20 \%$ y $17 \%$ ), Ecuador (20\%), Perú (19\%) y Venezuela $(20 \%)^{(23-27)}$.

En este trabajo, la frecuencia de pacientes con Clase III fue de un $16 \%$, resultado semejante al encontrado por Mafla (13\%), Cifuentes (17\%), Rodríguez (11\%), Castillo $(22 \%)$, Murrieta $(10 \%)$ y Medina $(15 \%)^{(22,24,26,27,29,30)}$. Thilander y Boeck reportan resultados muy inferiores, con un $3 \%$ y $2 \%$ respectivamente ${ }^{(23,28)}$.

La frecuencia total de pacientes con Mordida Cruzada en este estudio fue del $23 \%$, cifra bastante superior a los trabajos de Zeñil que encontró una prevalencia del $3.14 \%$, Keske-Kisulak un 7.5\%,Beraud un $11.3 \%$; Narayan un 7,26\% y Gutiérrez un $16 \%{ }^{(31-35)}$.

En cuanto a la Mordida Cruzada Anterior, en este estudio se ha identificado con una frecuencia del $9,8 \%$, cifra similar a la de Mato Gonzales ${ }^{(11)}$, que reporta una frecuencia del $9.2 \%$, y a la de Gutiérrez ${ }^{(35)}$, que reporta una frecuencia del $7,4 \%$. Pero superior a la de Zeñil ${ }^{(31)}$ que reportó una frecuencia del $2.12 \%$. De Freitas reporta una frecuencia más elevada a la hallada por este estudio, con una frecuencia del $18 \%{ }^{(36)}$.

En cuanto a la Mordida Cruzada Posterior se encontró una frecuencia total del $13 \%$, muy similar a la de Beraud que encontró que la prevalencia de mordida cruzada posterior fue de $11.3 \%{ }^{(33)}$. Los resultados de este estudio son superiores a los reportados por Zeñil ${ }^{(24)}$, que identificó un $1,02 \%$ de mordida cruzada posterior y Gutiérrez $^{(35)}$ que reportó un $6,5 \%$. Por otro lado, Freitas reporta cifras mayores a la del presente trabajo, con una prevalencia de mordida cruzada posterior del $27 \%{ }^{(36)}$.

Del $13 \%$ que presentó mordida cruzada posterior, el $61 \%$ fue unilateral y el $39 \%$ bilateral, demostrando que existe mayor porcentaje de adolescentes con esta patología solo en una hemiarcada. Esto coincide con Gandini ${ }^{(37)}$ queobservó15,5\% de escolares que presentaron mordida cruzada posterior unilateral y 7,5\% mordida cruzada bilateral; también con Beraud ${ }^{(33)}$, que encontró que la prevalencia de mordida cruzada posterior fue de $11.3 \%$; de los cuales $5.4 \%$ tenía mordida cruzada unilateral y $1.5 \%$ mordida cruzada bilateral.

Burgos $^{(38)}$ reporta la misma frecuencia para mordidas cruzadas posteriores uni y bilaterales ( $50 \%$ cada una), y también otros autores como Boeck ${ }^{(28)}$, reportan que hay una ligera mayor prevalencia de mordida cruzada posterior bilateral sobre la unilateral (Mordida cruzada bilateral, $12 \%$ y unilateral, $11.15 \%$ ); y Gutiérrez et. al. ${ }^{(35)}$, reportaron que del $42 \%$ de los pacientes que presentaron mordida cruzada posterior, $45 \%$ era unilateral y $55 \%$ bilateral. 
La frecuencia de Mordida en tijera en total fue del $5 \%$ (unilateral $4 \%$, bilateral $1 \%)$, cifra bastante elevada en comparación con Chacon (1\%), Boeck (1,9\%), Burgosy Peña que no la observaron. Esto demuestra una elevada frecuencia en la población de estudio sobre dicha maloclusión ${ }^{(21,28,38,39)}$.

La frecuencia de maloclusión en sentido sagital fue del $98 \%$, y en sentido transversal del $27,6 \%$.La maloclusión más frecuente en sentido sagital fue la clase I $(53 \%)$ seguida por la clase II (31\%) y la clase III (16\%). La frecuencia de Mordida Cruzada fue del $23 \%$, presentándose la mayoría de ellas en el sector posterior (57\%) y en forma unilateral (61\%).La frecuencia de mordida en tijera fue del $5 \%$, siendo el $4 \%$ de estas, unilateral. Se observa una importante frecuencia de maloclusiones en la población de estudio, la cual aún es posible revertir con éxito debido a la etapa de desarrollo en la que se encuentra dicha población. Estos datos son importantes para la Salud Publica y sirven como base para la implementación de programas preventivos, interceptivos o correctivos. Además, permitedeterminar la frecuencia con que estas patologías orales se manifiestan en las diferentes poblaciones, según recomendaciones dadas por la OMS.

\section{Conflicto de I nterés}

Los autores declaran no tener conflictos de interés.

\section{REFERENCI AS BIBLI OGRÁFI CAS}

1. Graber TM, Vanarsdall RL. Ortodoncia: principios y técnicas actuales. $4 \mathrm{a}$ ed. Madrid: Elsevier; 2006.

2. Proffit WR, Fields HW, Sarver DM. Ortodoncia contemporánea. 4a ed. Barcelona: Elsevier Mosby; 2008

3. Ugalde F. Clasificación de la maloclusión en los planos anteroposterior, vertical y transversal. Revista ADM. 2007; LXIV(3): 97-109

4. Vellini F. Ortodoncia Diagnóstico y Planificación Clínica. Sao Paulo: Editorial Artes Medicas Latinoamérica; 2002.

5. Castillo-Carmona I, Feregrino-Vejar L, Rojas-García A， Gutiérrez-Rojo J. Frecuencia de maloclusión en pacientes que acuden a atención ortodóncica en la zona centro de Tepic, Nayarit. Rev Tamé 2016; 5 (13): 452-4.

6. Pan American Health Organization. Health in the Americas. Washington DC: Paho; 1998. p. 413-27.

7. Pulgar J. Clasificación correlativa de Bimler y la clasificación de Angle: Estudio correlacional. Trabajo especial de grado para optar al título de especialista en ortopedia maxilar. [monografía en Internet] Maracaibo: Universidad de Zulia; 2009: 27-30 [acceso 13 de octubre de 2020]. Disponible en: https: // docplayer.es/82276627-

Clasificacion-correlativa-de-bimler-y-laclasificacion-de-angle-estudiocorrelacional.html
8. Canut J. Ortodoncia clínica y terapéutica. 2da ed. Barcelona: Editorial Masson; 2005.

9. Hernández J, Padilla M. Tratamiento temprano de la mordida cruzada anterior. Revisión de la literatura. Rev. Estomat. Univalle. 2011; 19(2): 40-7.

10. González G, Fuenmayor LM. Mordida Cruzada Anterior. Revisión Bibliográfica. Revista Latinoamericana de Ortodoncia y Odontopediatría.[Internet] 2012 [acceso 13 de octubre de 2020]; Disponible

en: https: //www. ortodoncia.ws/ publica ciones/2012/art-18/\#

11. Mato González A, Pérez Mendoza L, Rodríguez Fuego $M$, González Gutiérrez A. Rev. Ciencias Médicas de Pinar del Río. Julio-agosto, 2016; 20 (4) : 458-64.

12. Shu Ge Y, Liu J, Guo X, Han JL. A follow-up study of early interceptive treatment of primary anterior crossbites. European Journal of Orthodontics. 2011; 33(5):551-7.

13. Prakash $\mathrm{P}$, Durgesh $\mathrm{BH}$. Anterior Crossbite Correction in Early Mixed Dentition Period Using Catlan's Appliance: A Case Report. ISRN Dentistry [internet] 2011. [acceso 13 de octubre de 2020]; Disponible en: https://www.researchgate. net/publica tion/51710737_Anterior_Crossbite_Co rrection_in_Early_Mixed_Dentition_Per 
iod_Using_Catlan's_Appliance_A_Case _Report

14. Nanda R. The differential diagnosis and treatment of excessive overbite. Dent Clin North Am. 1981 Jan;25(1):69-84. PMID: 6937401.

15. Vadiakas PG, Michael WR. Primary posterior cross bite: diagnosis and treatment. Pediatr Dent. 1991; 16(1): 1-4.

16. Kerosuo $\mathrm{H}$. Occlussion in the primary and early mixed dentitions in a group of Tanzanian and Finnish children. J Dent Child. 1990; 57 (4): 293-8.

17. Ogaard B, Larsson E, Lindsten R. The effect of sucking habits, cohort, sex, intercanine arch widths, and breast or bottle feeding on posterior cross bite in Norwegian and Swedish 3-year-old children. Am J Orthod Dentofac Orthop. 1994; 106 (2): 161-6.

18. Koch G, Poulsen S. Pediatric dentistry. A clinical approach. 2nd ed. Oxford: Wiley-Blackwell; 2009.

19. Migale $D$, Barbato $E$, Bossù $M$, Ferro R, Ottolenghi I. Oral health and malocclusion in 10-to-11 years-old children in southern Italy. European Journal of Paediatric Dentistry. 2009; 10 (1): 13-8.

20. Angle E. Treatment of malocclusion of the teeth and fractures of the maxillae. Philadelphia:The S.S. White Dental Manufacturing Company; 1898.

21. Chacón P, Silva-Esteves JF. Tratamiento Temprano de la Mordida en Tijera Unilateral. Odontol Pediatr. 2015; 14 (2); 164-70.

22. Murrieta J, Cruz P. Prevalencia de maloclusiones dentales en un grupo de adolescentes mexicanos y su relación con la edad y el género. Acta Odont Venez 2007; 45: 74-8.

23. Thilander B, Pena L, Infante C, Parada $S$, de Mayorga C. Prevalence of malocclusion and orthodontic treatment need in children and adolescents in Bogotá, Colombia. An epidemiological study related to different stages of dental development. Eur Orthod J. 2001; 23(2): 153-67.

24. Mafla AC, Barrera DA, Muñoz GM. Malocclusion and orthodontic treatment need in adolescents from
Pasto, Colombia. Rev Fac Odontol Univ Antioq 2011; 22(2): 173-185.

25. FajardoV, González, C. Prevalencia de maloclusiones dentales en escolares de 12 años en Monay - Cuenca. Rev OACTIVA UC Cuenca. 2016; 1 (2): 238.

26. Rodríguez J. Prevalencia de maloclusión y necesidad de tratamiento ortodóntico en escolares de 12 años de instituciones educativas públicas-Trujillo 2015. Rev. Dspace. Unitru. 2016; 1(2): 1-6.

27. Medina C. Prevalencia de maloclusiones dentales en un grupo de pacientes pediátricos. Acta odontol. venez [Internet] 2010 [acceso 13 de octubre de 2020]; 48 (1) Disponible en: https://www.actaodontologica.com /ediciones/2010/1/art-10/\#

28. Boeck E, Pizzol K, Navarro N, Chiozzini $\mathrm{N}$, Rozato A. Prevalência de maloclusão em escolares de 5 a 12 anos de rede municipal de ensino de Araraquara. Rev. CEFAC. 2013 SetOut; 15(5): 1270-80.

29. Cifuentes C, Muñoz F, San Martin N, Oliva C. Necesidad de tratamiento ortodóncico según índice DAl y Angle en adolescentes de 1 a 13 años de la comuna de Curanilahue en el año 2014. Revista Int. J. Med. Surg. Sci., 2016; 3 (2):829- 37.

30. Castillo I, Feregrino L, Rojas A, Gutiérrez J. Frecuencia de maloclusión en pacientes que acuden a atención ortodóncica en la zona centro de Tepic, Nayarit. Rev Tamé 2016; 5 (13): 452-4.

31. Zeñil CE. Prevalencia de mordidas cruzadas en pacientes de cuatro a 12 años en la Unidad de Especialidades Odontológicas. Rev Sanid Milit 2005; 59(3): 165-70.

32. Keske-Nisula K, Lehto R, Lusa V. Occurrence of malocclusion and need of orthodontic treatment in early mixed dentition. Am J Orthod Dentofacial Orthop. 2003 Dec; 124(6): 631-8.

33. Beraud D, Sánchez M, Murrieta J, Mendoza V. Prevalencia y factores de riesgo de mordida cruzada posterior en niños de 4-9 años de edad en ciudad Nezahualcóyotl. Bol. Med. 
Hosp. Infant. Mex. 2004; 61 (2); 1418.

34. Narayanan $\mathrm{R}$, Jeseem $\mathrm{M}$, Kumar $\mathrm{T}$. Prevalence of maloclusión among 10 12 years old schoolchildren in Kozhikode district, Kerala: an epidemiological Study. Editorial International Journal Of Clinical Pediatric Dentistry, 2016; 9(1):50-5.

35. Gutiérrez $D$, Diaz $R$, Valenti $M$. Prevalencia de mordida cruzada dental en pacientes pediátricos de la clínica periférica "Las águilas" turno vespertino de octubre 2005 a marzo 2006. Revista Latinoamericana de Ortodoncia y Odontopediatría [Internet] 2007[acceso 13 de octubre de 2020]; Disponible en: https: //www. ortodoncia.ws/ publica ciones/2007/art-10/

36. De Freitas $M$, De Freitas $D$, De Sá L F. Prevalência das más oclusões em pacientes inscritos para tratamento ortodônticona faculdade de odontologia de bauru - usp. Rev Fac Odontol Bauru 2002; 10(3): 164-9.
37. Gandini MRS, Santos Pinto A, Gandini Junior LG, Martins JCR, Mendes AJD. Estudo da oclusão dentária de escolares da cidade de Araraquarana fase de dentadura mista. Ortodontia 1994 Sep./Dec; 27 (3): 37-49.

38. Burgos D. Prevalencia de maloclusiones en niños $y$ adolescentes de 6 a 15 años en Frutillar, Chile. Int. J. Odontostomat., 2014; 8(1): 13-9. b

39. Peña $M$, Rojas $M$, Tirado $A$, Benavides B, Hurtado $M$, Ruiz A. Prevalencia de la maloclusión en tres planos del espacio en pacientes diagnosticados con defectos del habla en las clínicas de la especialización de ortopedia funcional y ortodoncia de la Universidad Cooperativa de Colombia. Rev. estomatol. 2014; 22(1): 26-32. 